\title{
Study of morphological hysteresis in partially immiscible polymers
}

\author{
Carmela Tufano • Gerrit W. M. Peters • \\ Peter Van Puyvelde • Han E. H. Meijer
}

Received: 1 April 2008 / Accepted: 5 November 2008 / Published online: 10 December 2008

(C) The Author(s) 2008. This article is published with open access at Springerlink.com

\begin{abstract}
The morphology evolution of two systems of partially immiscible polymers, differing in miscibility, is investigated by means of rheological experiments and optical microscopy. For each system, two concentrations, $10 \%$ and $20 \%$, are used. For immiscible systems, a hysteresis zone, defined by coalescence and breakup, exists where the average drop radius is not a unique function of the shear rate. We investigate whether the findings also apply to partially immiscible polymers. The average radii at different shear rates, measured with rheology, are compared to model predictions. The hysteresis zone, if present, is indeed affected by the polymeric system, the concentration and the flow history applied. Coalescence evolution is measured for three different step-downs in shear rate. For both $10 \%$ systems, the resulting average radii show a rather high scattering and do not match the theoretical predictions. For the $20 \%$ concentrations, the average experimental drop sizes seem independent of the magnitude of the step-down, at least during a certain period of time. Thereafter, it experiences a sudden, in the time scale of the experiments unbounded, increase in size that is more pronounced for the higher step-downs. Deviations of the experimental data from theoretical predictions are attributed to the partially immiscible character of the systems, yielding enhanced coalescence which, in turn, can induce confinement effects.
\end{abstract}

C. Tufano · G. W. M. Peters $(\bowtie) \cdot H$. E. H. Meijer Eindhoven University of Technology,

Eindhoven, The Netherlands

e-mail: g.w.m.peters@tue.nl

P. Van Puyvelde

Katholieke Universiteit Leuven, Leuven, Belgium
Keywords Morphological hysteresis • Partially immiscible polymers .

Morphology evolution · Blend $\cdot$ Confined flow

\section{Introduction}

The steady-state morphology of blends obtained by mixing immiscible polymers has been found to be a unique function of the flow history applied, while preparing and processing the blend, and it is considered as the result of a dynamic equilibrium between two competing phenomena: breakup and coalescence (Takahashi et al. 1994; Vinckier et al. 1996). Both processes are determined by two dimensionless numbers: the capillary number $C a=\eta_{m} \dot{\gamma} R / \sigma$, with $\eta_{m}$ the viscosity of the matrix phase, $\dot{\gamma}$ the shear rate, $R$ the drop radius, and $\sigma$ the interfacial tension of the polymer pairs, and the viscosity ratio $p=\eta_{d} / \eta_{m}$, where $\eta_{d}$ is the viscosity of the dispersed phase. Grace (1982) devoted his life to determine how the critical capillary number, defined as the capillary number at which breakup of a single drop occurs, depends on the viscosity ratio, defining in shear and extensional flows the limiting condition for breakup to occur. Concerning the flowdriven coalescence limit for two droplets, Chesters (1991) proposed a probability of coalescence based on the interaction time during the collision of drops and the time needed to drain the matrix film trapped between them. Based on the mobility of the interface (fully mobile, partially mobile, and immobile), three different expressions to, for a given shear rate, calculate the maximum radius, above which coalescence does not occur anymore, were proposed. An overview of the physics behind breakup and coalescence is reported by 
Grmela et al. (2001). Also, some experimental studies on the effects of simple shear flow on the morphology evolution in immiscible polymer blends have been carried out. Elmendorp (1986) showed that the equilibrium between breakup and coalescence can be reached only for shear rates higher than the critical one, which is defined as the shear rate at which the theoretical limiting curves for breakup and coalescence cross. Grizzuti and Bifulco (1997), Vinckier et al. (1998), and Minale et al. (1997, 1998) showed, by means of rheological experiments and optical microscopy (OM), that the steady-state morphology is reached only after a certain critical shearing time. However, Janssen and Meijer (1995) reported that flow conditions might exist in which more that one morphology is possible. For shear rates lower than the critical one, the dynamic equilibrium between breakup and coalescence cannot be obtained, or, at least, it cannot be reached in a reasonable shearing time. In that case, there exists a pseudoequilibrium zone, in which the morphology depends on the initial conditions and neither breakup nor coalescence occurs. Minale et al. (1997) demonstrated the existence of this hysteresis zone with a model blend at fixed concentration, and they showed that, when the average drop radius in the blend is larger than the one predicted by coalescence limits and lower than the breakup model prediction at the same experimental shear rate, multiple steady-state morphologies, or pseudo steady-state morphologies, are reached. The final morphology was shown not only to depend on the characteristics of the blend components and history of flow but also on the initial conditions. The effects of viscosity ratio and concentration of the blend have been studied by Minale et al. (1998) for the same model system. They showed that the hysteresis region shifts to smaller shear rates and narrows with increasing the concentration of the dispersed phase. Apart from the aforementioned studies, several others can be found in the literature, all concerning the coalescence in immiscible polymer blends (Elmendorp and van der Vegt 1986; Fortelny and Kovar 1988; Rusu and PeuvrelDisdier 1999; Verdier and Brizard 2002).

In this study, we investigate the occurrence of a hysteresis zone and the flow-driven coalescence behavior for two partially immiscible polymer systems, having different diffusivity. The effect of the flow history applied, initial conditions, and concentration of the blends are investigated by means of rheological measurements and OM. The measured morphology evolution is compared to theories available in literature for breakup and coalescence. The use of dynamic measurements as an experimental morphology probing method is critically reexamined.

\section{Materials and methods}

\section{Materials}

The blends investigated are prepared using as disperse phase polybutene (PB, Indopol H-25, BP Chemicals, UK) and polybutadiene (PBD, Ricon 134, Sartomer) respectively, while polydimethylsiloxane (PDMS, UCT) is chosen as continuous phase. These three materials are liquid and transparent at room temperature, and they exhibit almost matched refractive indices, leading to a limited turbidity of the blends, which allows rheooptical experiments. The densities $(\rho)$ of the materials are measured at $23^{\circ} \mathrm{C}$ by means of a digital density meter (DMA 5000, Anton Paar) and, due to the small differences in the density values of the dispersed and continuous phases, buoyancy effects can be neglected given the time scale of the experiments. Zero shear viscosities $(\eta)$ are measured at $23^{\circ} \mathrm{C}$ using a rotational rheometer (Rheometrics, ARES) equipped with a parallel plate geometry and applying steady shear rates. The viscosity of the pure components is independent of shear rate in the whole range of shear rates applied. In addition, the first normal stress difference is too small to be measured with our equipment. The steady interfacial tensions $(\sigma)$ are measured at room temperature by means of a pendent drop apparatus (PAT-1, Profile Analysis Tensiometer, Sinterface, Germany). The average molecular weight $\left(M_{n}\right)$, densities and viscosities of the phases and the interfacial tensions of the two polymer pairs at room temperature are shown in Table 1. Two different concentrations $(10 \%$ and $20 \%$ mass fraction of $\mathrm{PB}$ and $\mathrm{PBD}$, respectively) are investigated and mixing is performed following the protocol proposed by Takahashi et al. (1994) and Vinckier et al. (1996).

\section{Experimental methods}

To erase influence of preparation and loading, all systems are preconditioned at the beginning of each experiment by shearing them for a time sufficiently long to obtain a steady state morphology. The total minimum strain required is determined in advance, using transient stress relaxation tests that are suitable for this purpose given their sensibility to the initial

Table 1 Selected model components

\begin{tabular}{|c|c|c|c|c|}
\hline $\begin{array}{l}\text { Sample } \\
\text { drop/matrix }\end{array}$ & $M_{n}$ & $\begin{array}{l}\rho \\
\mathrm{kg} / \mathrm{m}^{3}\end{array}$ & $\begin{array}{l}\eta \\
\mathrm{Pa} \cdot \mathrm{s}\end{array}$ & $\begin{array}{l}\sigma \\
\mathrm{mN} / \mathrm{m}\end{array}$ \\
\hline$\overline{\mathrm{PB} / \mathrm{PDMS}}$ & $635 / 62,700$ & $874 / 972$ & $3.7 / 10.9$ & $2.2 * 10^{-3}$ \\
\hline PBD/PDMS & $8,000 / 62,700$ & $891 / 972$ & 13.6/10.9 & $4.2 * 10^{-3}$ \\
\hline
\end{tabular}


morphology (Takahashi et al. 1994). For our systems, the strain required is found to be between 2,500 and 3,000. In all experiments, we used a stress-controlled rheometer (Rheometrics DSR) equipped with a cone and plate geometry (cone diameter $40 \mathrm{~mm}$ and cone angle $0.04 \mathrm{rad}$ ) and a Peltier element to control the temperature, set to $23^{\circ} \mathrm{C}$, with an accuracy of $\pm 0.1^{\circ} \mathrm{C}$. After preshearing, the flow is stopped and oscillatory tests are performed to obtain the elastic modulus at frequencies varying from 0.1 to $100 \mathrm{rad} / \mathrm{s}$. Subsequently, a new steady shear, at a different shear rate, is applied. Experiments are carried out with both increasing and decreasing shear rates. In the case where shear rates are decreased, drops coalesce and, therefore, the average radii measured in this way are expected to follow the predictions from coalescence theory. In the opposite case, breakup dominates and results are compared to the breakup predictions. When investigating the effect on coalescence of different step-downs in shear rate, the sample is first preconditioned at an initial shear rate of $8 \mathrm{~s}^{-1}$ for $1,250 \mathrm{~s}(\approx 10,000$ strain units). Next, the desired step-down in the shear rate is applied, whereafter the flow is stopped at different time intervals from the step-down to perform the dynamic tests that ultimately yield the average drop radii. Indeed, a small strain oscillatory flow does not affect the morphology (Graebling et al. 1993, 1994; Gramespacher and Meissner 1992; Vinckier et al. 1996). OM is performed in order to interpret some of the results and to check the actual drop size.

Morphology probing using dynamic measurements

For the pure components, the value of storage modulus $G^{\prime}$ is zero. The blends show a typical linear viscoelastic behavior, with a non-zero $G^{\prime}$, even at the smallest concentrations investigated. Figure 1, left, shows the elastic moduli of the blend measured upon cessation of the flow, after preshearing at a shear rate of 2 and $0.1 \mathrm{~s}^{-1}$ for 3,000 strain units, respectively, showing a shoulder at low frequencies, that accounts for a relaxation process that is due to a perturbation of the shape of the dispersed droplets during the oscillatory flow. Different preshear rates result in different morphologies yielding different elastic moduli. Lowering the preshear rate makes the shoulder move to lower frequencies, indicating that larger droplets form at these lower shear rates.

To find the average drop diameter in the blend from dynamic measurements, the continuous relaxation spectrum is calculated from the dynamic moduli using a nonlinear regression program (Honerkamp and Weese 1993); see Fig. 1, right. The initial part of the spectra is identical and it reflects the contribution of the pure components. We are interested in the relaxation time $\tau$ corresponding to the peak, which is characteristic for drop relaxation. The average droplet size $R$ can be calculated from this relaxation $\tau$ using an approximate equation derived from the emulsion model of Palierne (1990):

$\tau=\frac{\eta_{m} R}{4 \sigma} \frac{(19 p+16)(2 p+3-2 \phi(p+1))}{10(p+1)-2 \phi(5 p+2)}$

where $p$ is the viscosity ratio $\left(p=\eta_{d} / \eta_{m}\right.$, with $\eta_{d}$ as the viscosity of the dispersed phase and $\eta_{m}$ as the viscosity of the continuous phase), $\phi$ is the volume fraction, and $\sigma$ is the interfacial tension. Although the value of $\tau$ can be directly obtained from the relaxation spectrum,
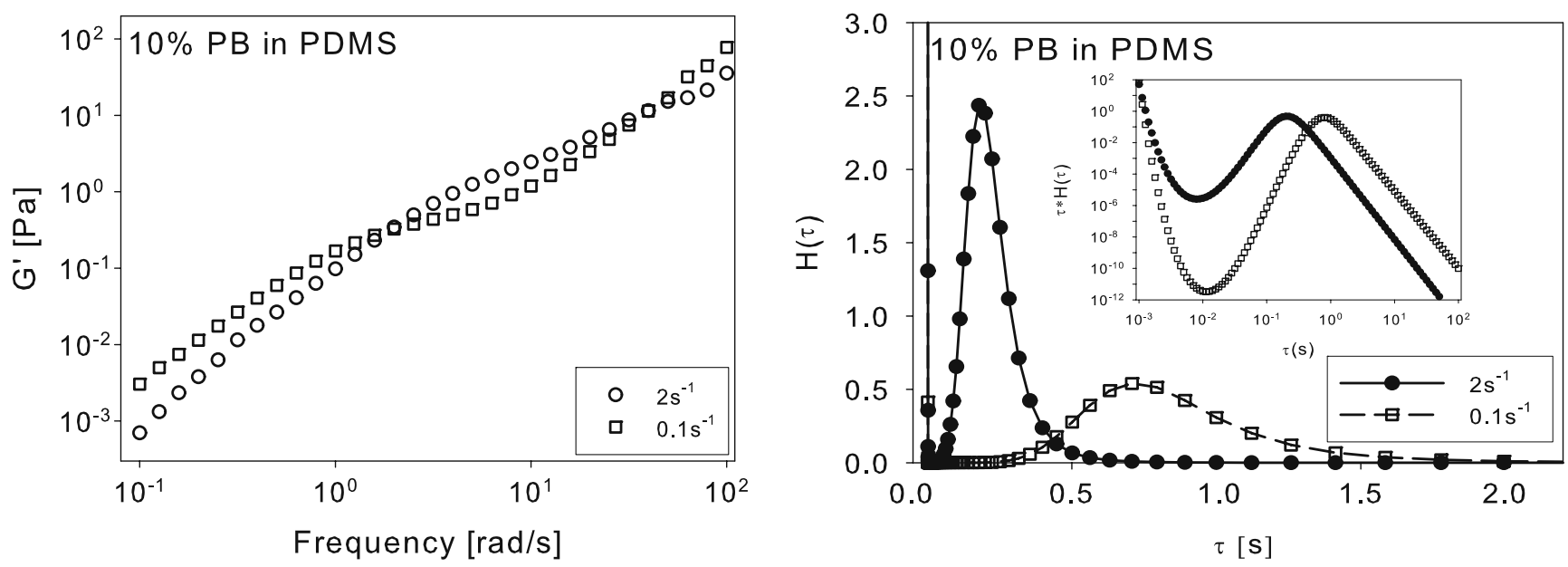

Fig. 1 Storage moduli (left), relaxation spectra and weighted relaxation spectra (inset plot) (right) upon cessation of flow after shearing at $\dot{\gamma}=2 \mathrm{~s}^{-1}$ and $\dot{\gamma}=0.1 \mathrm{~s}^{-1}$ for 3,000 strain units for a 10-wt.\% PB in PDMS blend 
as the time at which $H(\tau)$ reaches the maximum, a different approach was proposed by Gramespacher and Meissner (1992). They suggested to plot the weighted time relaxation spectrum, $\tau * H(\tau)$ vs $\tau$ and to use the time at which this curve reaches the maximum as the relaxation time of the drops. The use of the first moment of the relaxation spectrum amplifies the contribution of slower processes, thus enhancing effects of the interfacial relaxation process. The inset plot in Fig. 1, right, shows the weighted relaxation spectra at the two shear rates considered. In both procedures, a longer relaxation time and a lower maximum is found for the lower shear rate. While the relaxation time is linked to the average size of the inclusions, the magnitude of the maximum in the relaxation spectrum, as well as in the weighted relaxation spectrum, contains information on the amount of interfacial area. Decreasing the shear rate, coalescence is promoted and, due to the constant fraction of dispersed phase, the morphology evolution will lead to less and larger drops, which require longer times to relax; see Eq. 1. Fewer large drops imply, on the other hand, less interfacial area, which reduces the magnitude of the maximum; see Fig. 1, right. Figure 2 shows the average droplet radii for the 10\% PB/PDMS blend calculated using Eq. 1 with the relaxation time retrieved from the time relaxation spectra and from the weighted time relaxation spectra, respectively. Experiments are performed while decreasing the preshear rate. Clearly, both procedures give similar results (within experimental error), indicating that the average radii obtained are a good indication of the blend morphology. However, we experienced that, for the weighted relaxation spectra, the ampli-

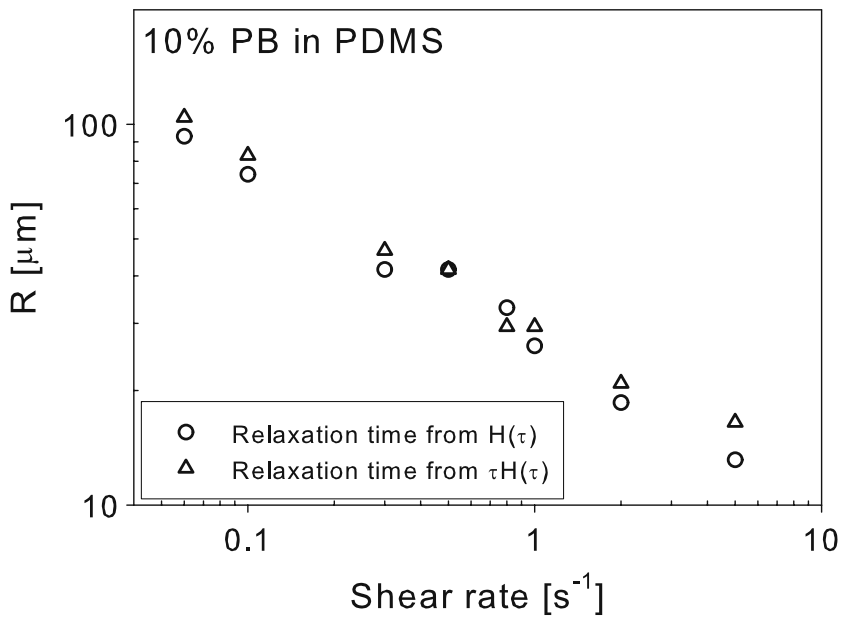

Fig. 2 Calculated average radii (Eq. 1), 10 wt.\% PB in PDMS blend, for decreasing shear rate. Radii from relaxation time spectra (circles) and weighted relaxation time spectra (triangles) fication of the slow relaxation phenomena was often more clearly present, which makes the analysis more precise. Therefore, this procedure is used. Vinckier et al. (1996) proposed a further improved approach, based on the assumption that the contribution of the different relaxation processes involved are additive. The time relaxation spectra of the pure components, weighted by their volume fraction, were subtracted from the blend time relaxation spectrum, influencing the position of the maximum in $H(\tau)$ and, therefore, the predicted average radius. However, since, in our cases, the relaxation of the pure components is much faster than that of the interface, these corrections will not have to be used here.

\section{Hysteresis zone}

Theories to probe morphological hysteresis

The morphology resulting from dynamic experiments is compared to breakup and coalescence theories. At a fixed shear rate, the flow is able to breakup drops with sizes above a critical value, whereas drops with smaller radii will collide and eventually coalesce. Grace (1982) experimentally found the critical size of a droplet, for a fixed shear rate, above which breakup occurs and below which coalescence dominates. Based on Grace's experimental results, Bruijn (1989) suggested a fitted curve:

$$
\begin{aligned}
\log \left(\frac{\eta_{m} \dot{\gamma} R}{\sigma}\right)= & -0.506-0.0994 \log (p)+0.124 \log ^{2}(p) \\
& -\frac{0.115}{\log (p)-\log \left(p_{\mathrm{cr}}\right)},
\end{aligned}
$$

where $p_{\text {cr }}=4.08$ is the viscosity ratio above which breakup is not possible anymore, at least in a start-up shear flow, $\dot{\gamma}$ is the shear rate, and $R$ is the average drop radius in the blend. Theories and numerical models support the experimental data (Jackson and Tucker 2003; Taylor 1932; Yu et al. 2002, 2005). Also, the coalescence process has been modeled and different mobilities of the sharp interfaces (from mobile to partially mobile and fully immobile) are distinct, greatly influencing the drainage rate. Also, the more physical diffuse interfaces have obtained a lot of attention (via diffuse interface modeling), and while, for breakup, excellent results are obtained, the spatial resolution of the mesh makes coalescence events occur too fast (Anderson et al. 1998; Cahn and Hilliard 1958, 1965). The drainage model for sharp interfaces is chosen here. The maximum radius below which coalescence occurs 
is estimated for the case of a immobile interface (IM) and partially mobile interface (PM) (Chesters 1991):

IM $\quad R=\left(\frac{32}{9}\right)^{1 / 4} \cdot\left(\frac{h_{\mathrm{cr}}^{1 / 2} p}{\eta_{m} \cdot \dot{\gamma}}\right)^{-1 / 2}$

$\mathrm{PM} \quad R=\left(\frac{4}{\sqrt{3}} \frac{h_{\mathrm{cr}}}{p}\right)^{2 / 5} \cdot\left(\frac{\sigma}{\eta_{m} \cdot \dot{\gamma}}\right)^{3 / 5}$,

where $h_{\text {cr }}$ is the critical film thickness. The maximum drop radius above which coalescence does not occur is a function of the coalescence conditions, the viscosity ratio $p$, and the interfacial tension. In the original paper of Mackay and Mason (1963), the drainage time refers to a droplet coalescing on a flat surface while, in our experiments, coalescence occurs between two droplets. To account for that, Eq. 3 is corrected by a factor 4 . The main difficulty is to find an adequate value for the critical film thickness of the matrix trapped between two coalescing drops, $h_{\text {cr }}$. A possibility is to calculated this parameter, e.g., using the formulation suggested by Chesters (1991):

$h_{\mathrm{cr}} \approx\left(\frac{\mathrm{AR}}{8 \pi \sigma}\right)^{1 / 3}$

where $A$ is the Hamaker constant. Since theories are derived for isolated pairs of droplets, while we are dealing with concentrated blends, some authors choose to use the $h_{\text {cr }}$ as an adjustable parameter. It then contains all the uncertainties and approximations of the model and, to a certain extent, it is a function of the concentration (Minale et al. 1997, 1998). We will use a
Table 2 Critical film thickness $h_{\text {cr }}$ calculated with Eq. 5 for the $\mathrm{PB} / \mathrm{PDMS}$ and PBD/PDMS systems, for three different average drop radii $R$

\begin{tabular}{lll}
\hline$R[\mathrm{~m}]$ & $h_{\mathrm{cr}}^{P B}[\mathrm{~m}]$ & $h_{\mathrm{cr}}^{P B D}[\mathrm{~m}]$ \\
\hline $5 * 10^{-6}$ & $1 * 10^{-8}$ & $0.8 * 10^{-8}$ \\
$50 * 10^{-6}$ & $2 * 10^{-8}$ & $1.7 * 10^{-8}$ \\
$150 * 10^{-6}$ & $3 * 10^{-8}$ & $2.5 * 10^{-8}$ \\
\hline
\end{tabular}

fixed value for each system based on Eq. 5 and use only the predictions of the PM model.

\section{Hysteresis results}

To draw the coalescence line to theoretically bound the hysteresis zone, a value for the critical film thickness, $h_{\mathrm{cr}}$, which changes with the radius (see Eq. 5), is required. To choose, sensitivity analysis is performed. The average radii observed in our hysteresis experiments mostly range between $5 * 10^{-6}$ and $150 * 10^{-6} \mathrm{~m}$ for both blends, which, according to Eq. 5, lead to the $h_{\text {cr }}$ values shown in Table 2. The variations of the average drop radius, predicted by the PM model (Eq. 4) using these $h_{\text {cr }}$ values, are small only, see Fig. 3, which basically allow us to use a constant, concentrationindependent value of $h_{\text {cr }}$ for each of the two systems. We choose to use the values corresponding to the average radius of $50 * 10^{-6} \mathrm{~m}$ to investigate the existence and size of the hysteresis zone.

\section{$P B$ in $P D M S$}

The average radii measured for the 10 -wt.\% $\mathrm{PB}$ in PDMS system, at increasing and decreasing shear rates,
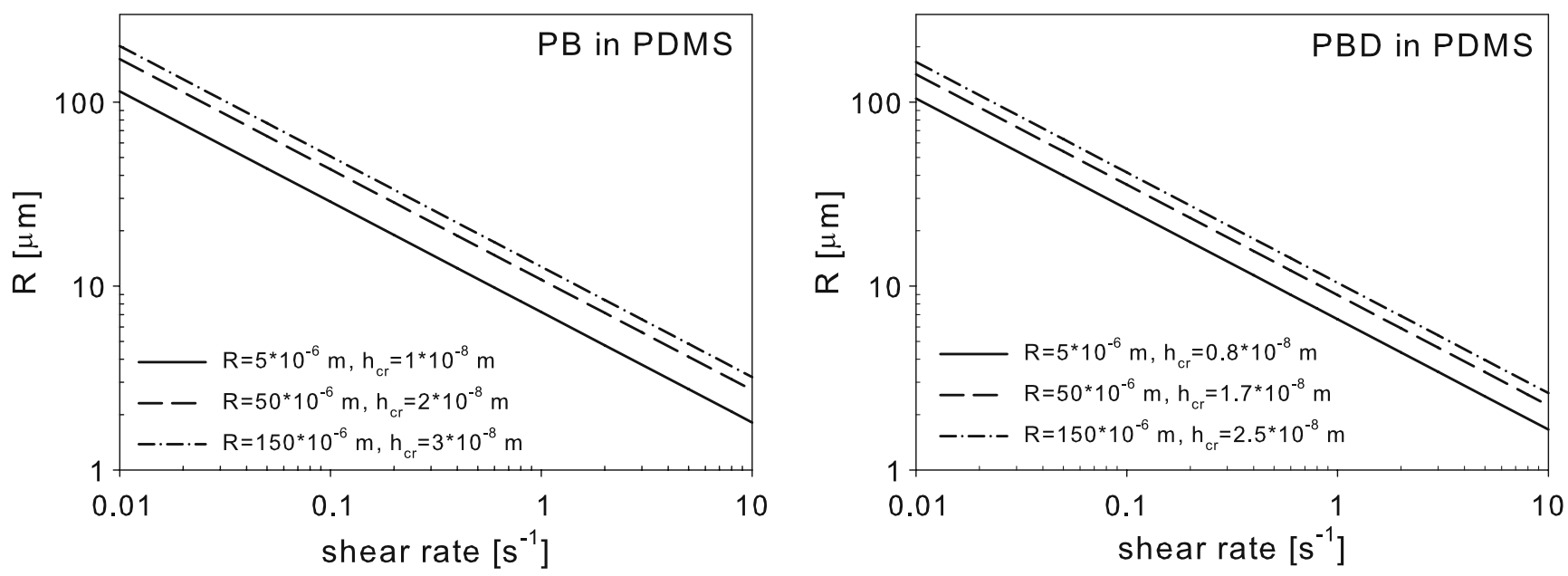

Fig. 3 Influence of different radii and the corresponding $h_{\mathrm{cr}}$ values (Eq. 5) on the average radii calculated with the PM model (Eq. 4) for the PB/PDMS system (left) and PBD/PDMS system (right) 
are shown in Fig. 4 (top) and compared to the model predictions for breakup (solid line) and coalescence (dashed line). At the lowest shear rate, the morphology is independent of the flow history applied; the same pseudo steady-state value is found for decreasing and increasing shear rates. We use $h_{\mathrm{cr}}=2 * 10^{-8} \mathrm{~m}$ (Eq. 5), which is larger than the one reported by Tufano et al. (2008b) for the same blend, but in that work, with a concentration of $1 \%, h_{\mathrm{cr}}=0.36 * 10^{-8} \mathrm{~m}$. Increasing $h_{\mathrm{cr}}$ values with increasing concentration were also reported in Minale et al. $(1997,1998)$ and two possible explanations were given: (a) the influence of dust particles
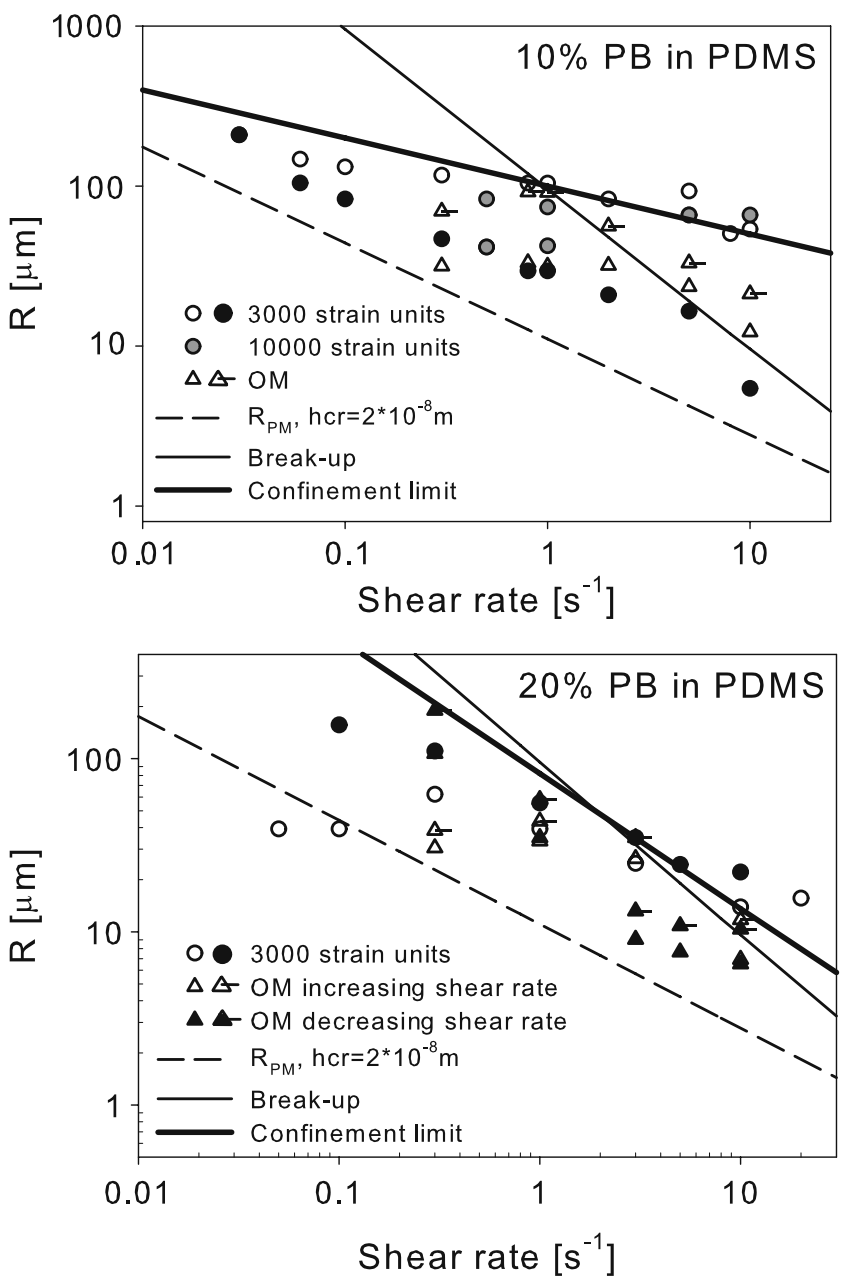

Fig. 4 Average radii obtained by using Eq. 1 and relaxation times from weighted relaxation spectra, after shearing for 3,000 (circles) and 10,000 (gray filled circles) strain units for the $10 \%$ $\mathrm{PB} / \mathrm{PDMS}$ system (top) and after shearing for 3,000 (circle) strain units for the $20 \%$ PB/PDMS system (bottom). Results obtained while increasing (open symbols) and decreasing (filled symbols) preshear are shown. Triangles are from OM results. The theoretical limiting curves for breakup (solid line, Eq. 2), coalescence using the PM model (dashed line, Eqs. 5 and 4) and confinement effects (thick solid line) are shown present in the blend or (b) the theory behind Eq. 4 is derived for a single pair of drops and not for more concentrated systems, where a coalescence event between two drops influences those in neighboring drops (Janssen 1993), and collisions between drops are more frequent and last longer than for two isolated drops meeting in a shear flow. However, according to the model behind Eq. 4, the time available for the drainage is proportional to $\dot{\gamma}^{-1}$, which is fixed for a given flow. By accounting for concentration effects through larger $h_{\text {cr }}$ values, the critical film thickness $h_{\text {cr }}$ looses its physical meaning. It becomes a fitting parameter only and contains all the uncertainty in the model and the effects of the concentration.

Results in Fig. 4 (top) show that, at shear rates lower than the critical one (the shear rate at which the breakup and coalescence curves cross), the hysteresis zone observed is not according to the theoretical expectations. For a decreasing shear rate, the experimental coalescence results run parallel to the theoretical curve, but the radii are larger. With increasing shear rate, instead of just crossing the hysteresis zone and following the breakup line, the drop radius is decreasing with a seemingly constant slope and the results extend beyond the breakup line. For the PB-PDMS system, it is, unfortunately, not possible to shear the sample at shear rates higher than the critical one due to the occurrence of shear fracture. To check whether the morphology evolution depends on the strain units of shear, the same experiments are repeated, shearing the blend for 10,000 strain units. The results are in good agreement with the data for 3,000 strain units, indicating that, in this case, there is no influence of the shearing time on the average radii. For a second check on the question of whether the deviation from theory is real, the same flow history is applied in OM experiments. While the rheological measurements are conducted with a cone and plate geometry, the OM measurements are performed with a plate-plate configuration. The gap between the plates is set to $400 \mu \mathrm{m}$ to limit the occurrence of confinement effects (Tufano et al. 2008a). $\mathrm{OM}$ pictures acquired at three different shear rates are shown in Fig. 5. The average radii (triangles) and the largest drop radius (signed triangles) resulting from the OM experiments are also plotted in Fig. 4.

The morphology depicted in Fig. 5 shows large droplets among much smaller ones, especially at the lower shear rates; the droplet distribution seems to be bimodal. At higher shear rates, the drop radius distribution becomes more uniform. Since in Fig. 4 the largest drop radii show the same trend as those obtained from rheology (although they are smaller), it follows that the rheological data mostly account for the relaxation times 

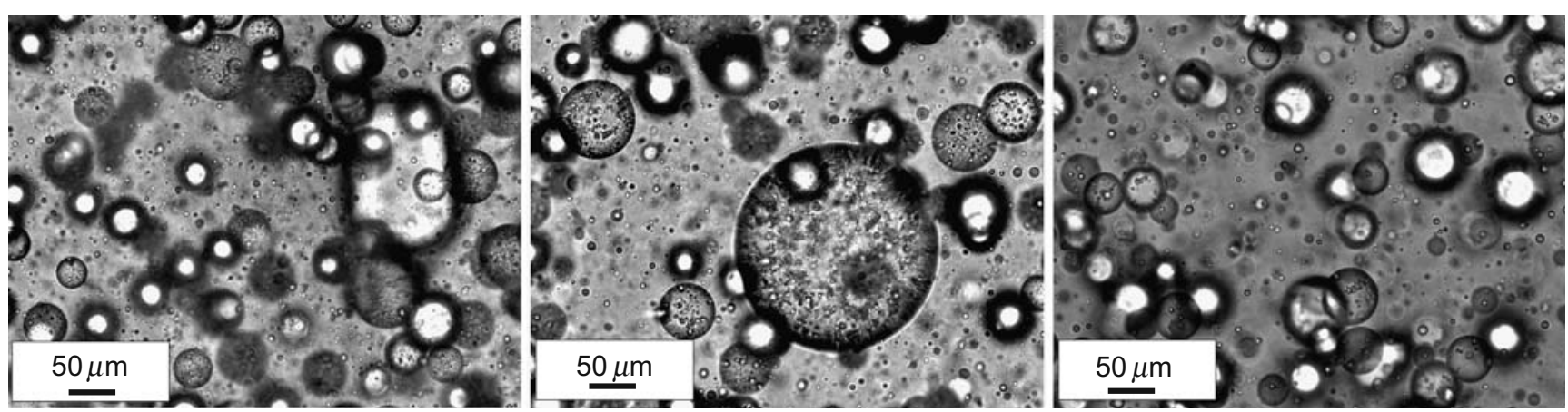

Fig. 5 Morphology for the 10-wt. \% PB in PDMS blend at shear rates $0.3,0.8$, and $5 \mathrm{~s}^{-1}$, increasing the shear rate

of the largest droplets, giving an estimated average radius in the blend larger than the effective one.

The data for the $20 \% \mathrm{~PB} / \mathrm{PDMS}$ blend are rather scattered [see Fig. 4 (bottom)] and the results become even more complicated for this higher concentration. A narrowing of the hysteresis zone is observed, in accordance with Minale et al. (1998). The data for increasing and decreasing shear rates almost coincide (within experimental error), i.e., the morphology seems to become independent of the flow history. However, similar phenomena as for the 10\% PB/PDMS blend are observed; drop radii are found that are above the breakup line and large droplets, which will excessively contribute to the rheology (yielding an overestimation of the average radius), are also found with OM (Fig. 6). In contrast to a number of published results, we do not find a good agreement between theory and average radius from dynamic measurements. Although our results are somewhat scattered, trends are clear. In an attempt to interpret them, two effects are considered that might help their explanation: (1) for partially immiscible blends, coalescence is drastically increased (Zdravkov et al.2006), which leads to large drops after a step-down in shear rate, (2) on the other hand, confinement of flows can cause phenomena like droplet ordering, string formation, etc. (Migler 2001; Pathak et al. 2002; Pathak and Migler 2003; Tufano et al. 2008a; Vananroye et al. 2006). Large drops, as observed with OM, easier feel the presence of walls. This influences, in a yet unpredictable manner, the rheological results. For a cone-plate geometry, where the gap varies from zero to maximum at the edge, part of the flow can be confined, e.g., in the area of flow where drops have the same characteristic size as the gap. The size of this confined region depends on both the blend system and the flow conditions. For a given shear rate, for the four blends studied, the degree of confinement $C_{d}=2 R / H$, with $R$, the drop radius, and $H$, the gap spacing between the parallel plates, at which wall effects are present, was measured by Tufano et al. (2008a) for different shear rates applied. A power law relation follows:

$C_{d}=C_{d 0} \dot{\gamma}^{-a}$

Figure 7 shows the measured values of $C_{d}$ for all four blend systems combined with the corresponding fits, using Eq. 6, and the fit parameters $C_{d 0}$ and $a$ in Table 3. The values of the prefactor $C_{d 0}$ are rather
Fig. 6 Morphology for the 20-wt.\% PB in PDMS blend at shear rates 1 and $0.3 \mathrm{~s}^{-1}$, decreasing the shear rate
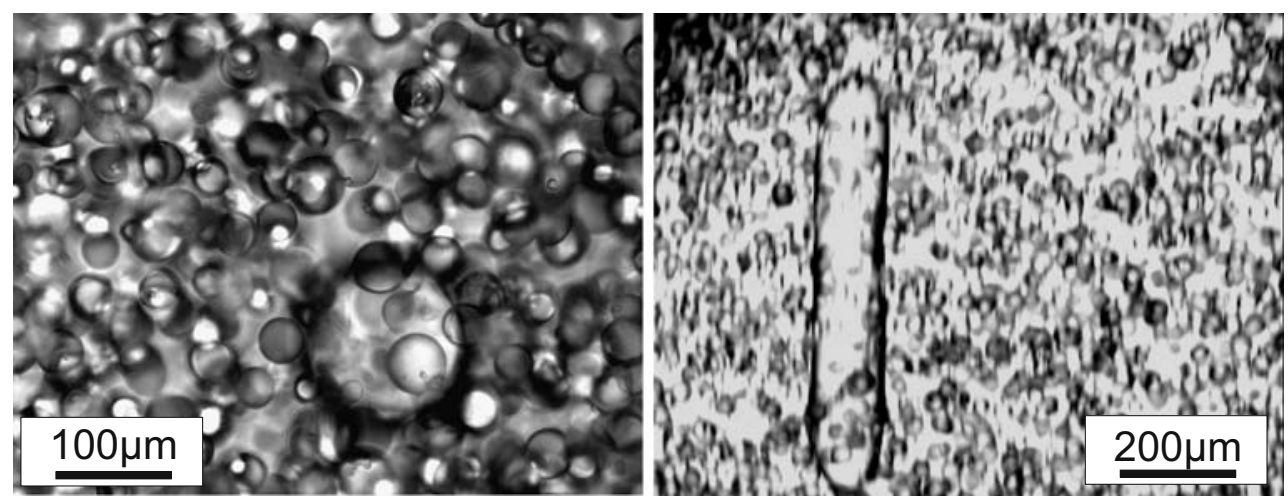


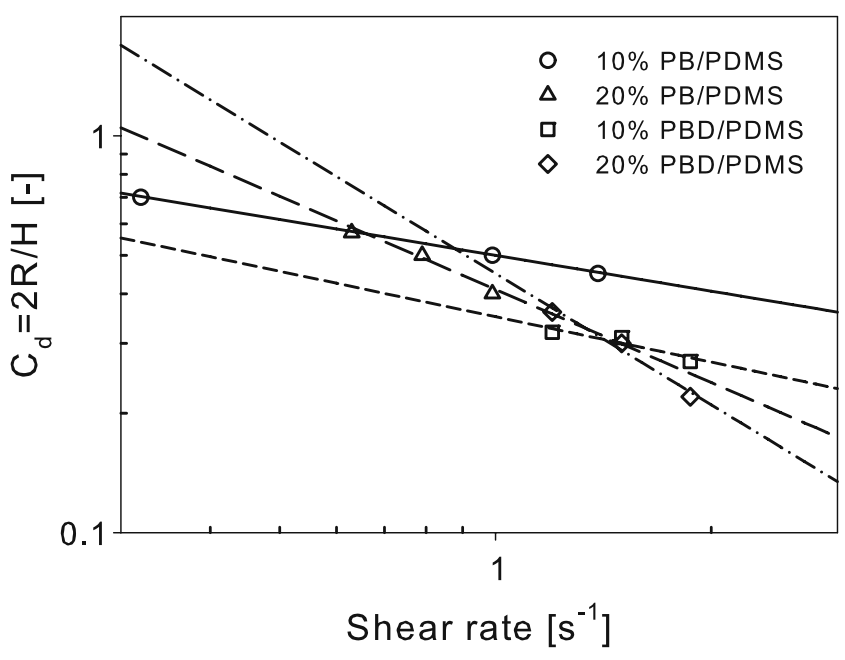

Fig. 7 Critical degree of confinements vs shear rate. Experimental results from Tufano et al. (2008a) (symbols) and fits using Eq. 6 (lines). The values for $C_{d 0}$ and $a$ are given in Table 3

close while the values of the exponent increase with viscosity ratio $p$ and with concentration. It is tempting to (try to) create a master curve, but this is outside the scope of this work and more data are needed. For a given drop size, we can deduce the sample volume that is prone to confined flow. For a drop size of $100 \mu \mathrm{m}$, as found for the $10 \% \mathrm{~PB} / \mathrm{PDMS}$ blend, confinement is expected up to half way the maximum cone radius (i.e., $25 \%$ of the sample area is confined or $10 \%$ of the torque is affected). Using the half-way gap height and the parameter sets of Table 3 and Eq. 6, we can draw confinement lines $R(\dot{\gamma})$ in Fig. 4 (and Fig. 8 in the next section for the PBD/PDMS blends). From these considerations, it seems reasonable to expect confinement effects during the dynamic measurements on partially immiscible polymers. Despite that, it is yet impossible to predict what effects will occur since confinement leads to complex, transient phenomena. While the build-up time for confined dominated morphologies typically takes a period in the order of minutes, the stationary state of morphology is often obtained only after a few hours (Migler 2001; Pathak et al. 2002; Pathak and Migler 2003; Tufano et al. 2008a; Vananroye et al. 2006). Therefore, the transient coalescence process gets

Table $3 \quad C_{d 0}$ and $a$ values from Eq. 6 for the four blends investigated

\begin{tabular}{lll}
\hline Blend & $C_{d 0}$ & $a$ \\
\hline $10 \%$ PB/PDMS & 0.5 & 0.3 \\
$20 \%$ PB/PDMS & 0.41 & 0.78 \\
$10 \%$ PBD/PDMS & 0.5 & 0.38 \\
$20 \%$ PBD/PDMS & 0.45 & 1.1 \\
\hline
\end{tabular}
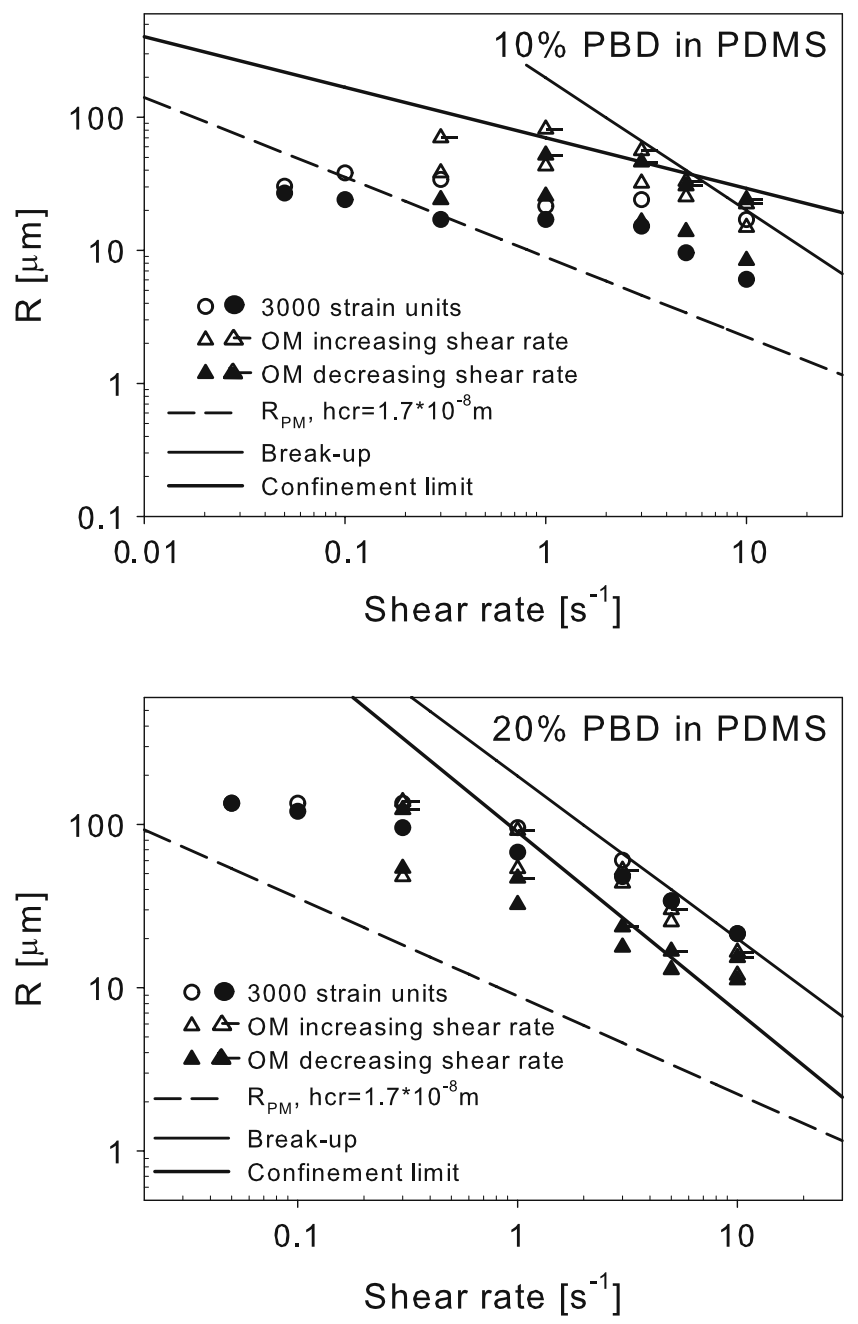

Fig. 8 As Fig. 4, now for 10\% (top) and 20\% (bottom) PBD in PDMS systems

coupled with effects of transient confinement. When confinement occurs, typically a mixture of standard and extended drops, or the opposite small drops, is formed, which causes an increased relaxation time (apparently larger drops) for the first case and a decreased relaxation time (apparently smaller drops) for the second case. During dynamic measurements, part of the structures extend to threads that subsequently breakup, leading to a bimodal distribution of drop size; the standard drops and the new drops from thread breakup. However, the thread-like structures can also survive for long periods of time, depending on the local degree of confinement (Son et al. 2003). As an example, for the $10 \% \mathrm{~PB} / \mathrm{PDMS}$ blend, it is known that partial miscibility is present between the phases that enhances the coalescence process dramatically (Zdravkov et al. 2006); larger droplets are formed quicker, strengthening the confinement. For the 20\% PB/PDMS blend 

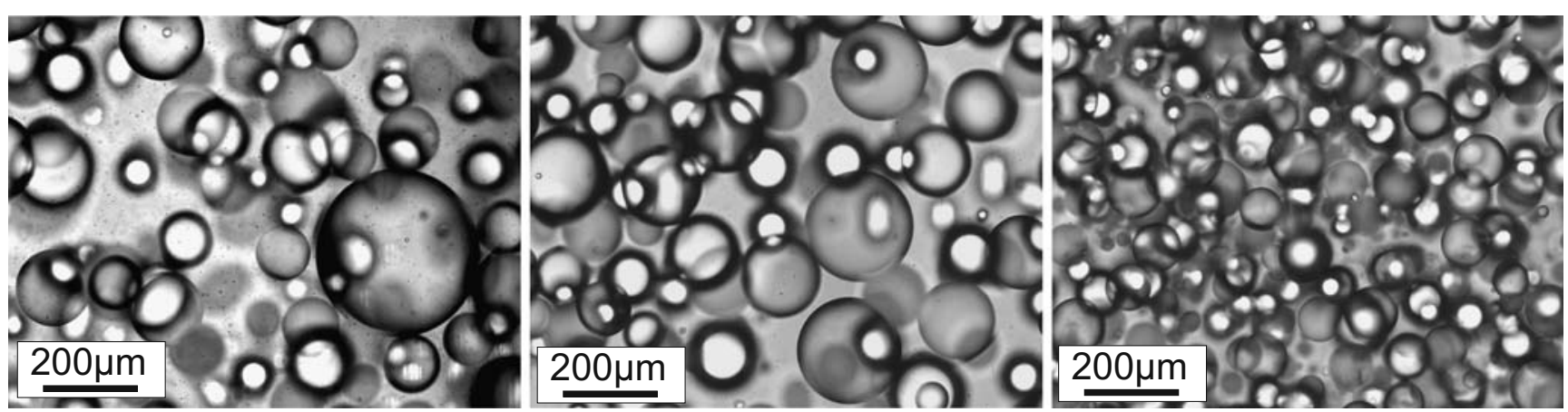

Fig. 9 Morphology for the 20-wt.\% PBD in PDMS blend at shear rate of, from left to right, 0.3, 1, and $3 \mathrm{~s}^{-1}$

(see Fig. 4, bottom) we expect confinement effects over the whole range of shear rates applied, and indeed, we have to question the reliability of the rheological results in this case. These considerations lead to the conclusions that, especially for the $10 \%$ PB/PDMS system, the OM results are in reasonable agreement with theory, considering the breakup line, while the dynamic results are not. Only when rheology and OM results match can confinement effects apparently be neglected, and rheometry could be a useful method to investigate blend morphology.

\section{$P B D$ in $P D M S$}

Similar to the 10 wt.\% PB in PDMS system, also for the PBD in PDMS blends, it is not possible to start experiments at shear rates higher than a critical one, due to the occurrence of shear fracture. The range of shear rates investigated is $0.05-10 \mathrm{~s}^{-1}$, see Fig. 8 (top), where the coalescence line is calculated with $h_{\mathrm{cr}}=1.7 * 10^{-8} \mathrm{~m}$ (the value corresponding to the same average value of $R=50 * 10^{-6} \mathrm{~m}$ as used for the PB/PDMS system). Also, for this blend, the drops seem to coalesce much easier than predicted by the theory and the average radii are always far above the coalescence model predictions. This again leads to a hysteresis zone narrower than expected from modeling. Both flow histories are repeated with OM. The results quantitatively confirm the data obtained with rheological experiments. Compared to the PB/PDMS results, the confinement line is mostly above the experimental results for the $10 \%$ blend; therefore, less influence of confinement is expected, while for the $20 \%$ blend, see Fig. 8, bottom, confinement plays a more pronounced role.

In line with the results for PB/PDMS, where an increase in the dispersed phase concentration narrows the hysteresis region, see also Minale et al. (1997,
1998), also for the 20\% PBD/PDMS system a narrow hysteresis zone is found. For an increasing shear rate, the average radii from $\mathrm{OM}$ are lower than the average radii obtained by rheological measurements. However, comparing the radii of the large drops from OM (Fig. 9) at each shear rate, with the data from rheology, it is concluded that rheology accounts mainly for larger drop radii, again overestimating the average drop radius. When decreasing the shear rate, the average radii measured with $\mathrm{OM}$ are found on a line parallel to the coalescence line and are smaller than the ones obtained with rheology.

\section{Coalescence after a step-down in shear rate}

Next, we investigate the morphology evolution after a step-down in shear rate in order to find out whether deviations from theory observed in the hysteresis zone are also reflected in the transient coalescence process. The experimental results are compared to theories for sharp interfaces. Experiments start from the same initial shear rate of $8 \mathrm{~s}^{-1}$, shearing for 10,000 strain units. Next, step downs of $1 / 40,1 / 10$, and $1 / 4$ are applied, reducing the shear rates to $0.2,0.8$, and $2 \mathrm{~s}^{-1}$, respectively.

\section{Modeling coalescence}

Chesters (1991) summarized the theory behind coalescence. Two characteristic times control the coalescence process; the interaction time between colliding drops, $t_{\text {int }}$, and the time needed to drain the matrix film trapped between the colliding drops, $t_{\text {drain }}$. Coalescence does not occur when the $t_{\text {drain }}$ is longer than $t_{\text {int }}$. The rate of change of the interfacial area, $Q$, in the case of monodisperse blends, is given by:

$$
\frac{d Q(t)}{d t}=C(t) P(t) \Delta S(t)
$$


where $C(t)$ is the collision frequency per unit volume, $P(t)$ is the fraction of collisions that leads to coalescence, i.e., the coalescence probability, and $\Delta S(t)$ is the variation in the interfacial area associate to a single coalescence event. To find expressions for these terms, we follow Chesters (1991). First, it is assumed that droplets move affine with the macroscopic flow, yielding a collision frequency per unit volume expressed as (Von Smoluchowski 1917):

$C(t)=\frac{2}{3} \dot{\gamma} D(t)^{3} n(t)^{2}$,

where $\dot{\gamma}$ is the shear rate, $D(t)$ is the drop diameter, and $n(t)$ is the number of drops per unit volume. In
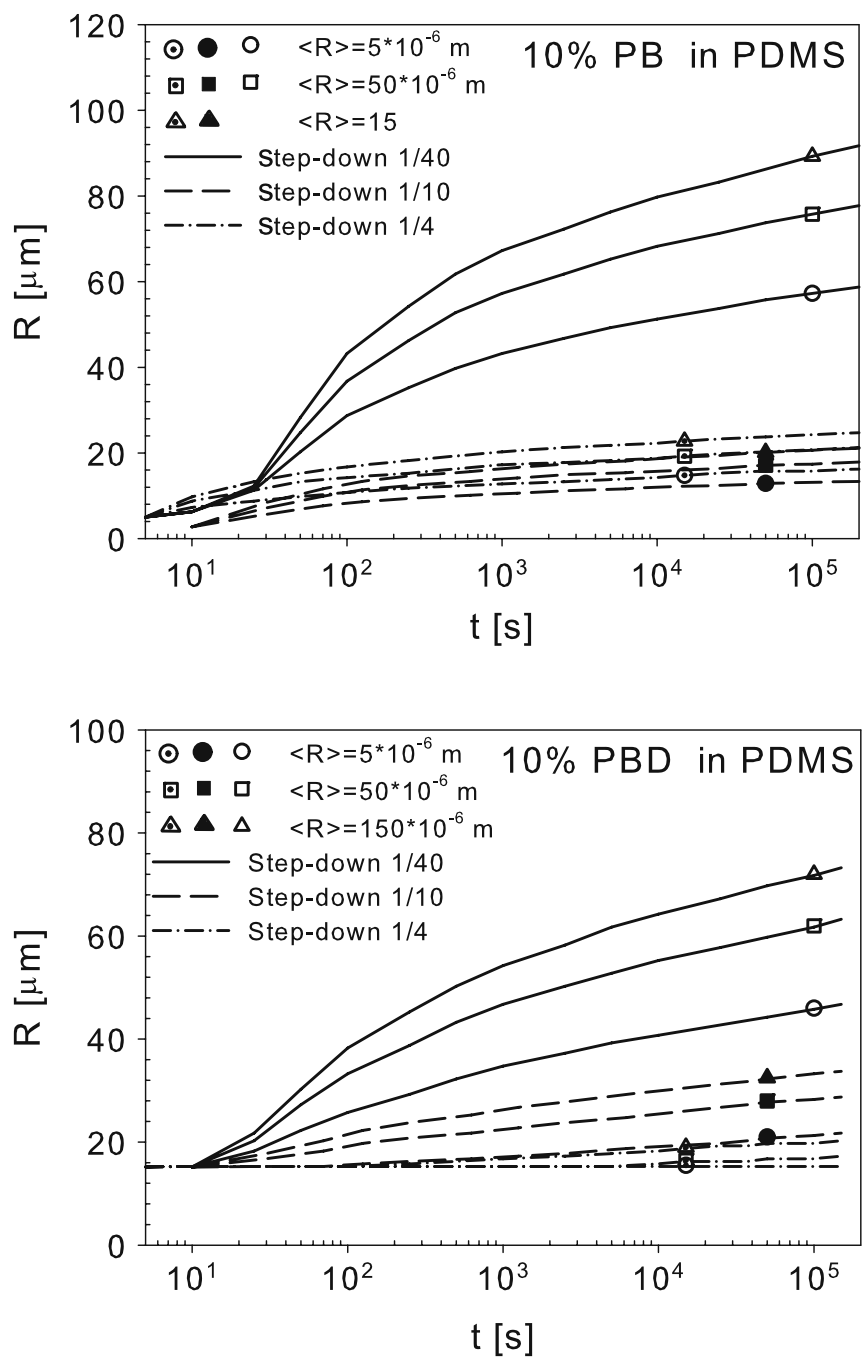

Fig. 10 Influence of different radii, and the corresponding $h_{\mathrm{cr}}$ values (Eq. 5) on the transient average radii calculated with the PM model (Eq. 4) after step-down in shear rate of $1 / 40$ (solid line), 1/10 (dashed line), and 1/4 (dash-dotted line) for the
Eq. 8, hydrodynamic interactions have been neglected; hence, it applies only to relatively dilute blends. The coalescence probability is given by:

$P(t)=\exp \left(-\frac{t_{\text {drain }}}{t_{\text {int }}}\right)$

where $t_{\text {int }}$ is assumed proportional to $\dot{\gamma}^{-1}$. Using an approximation for $t_{\text {drain }}$ in the case of a partially mobile interface leads to [for more details on deriving this expression, see Janssen (1993)]:

$P(t) \simeq \exp \left[-0.0765 \frac{p}{h_{\mathrm{cr}}}\left(\frac{\eta_{m} \dot{\gamma}}{\sigma}\right)^{3 / 2} D(t)^{5 / 2}\right]$
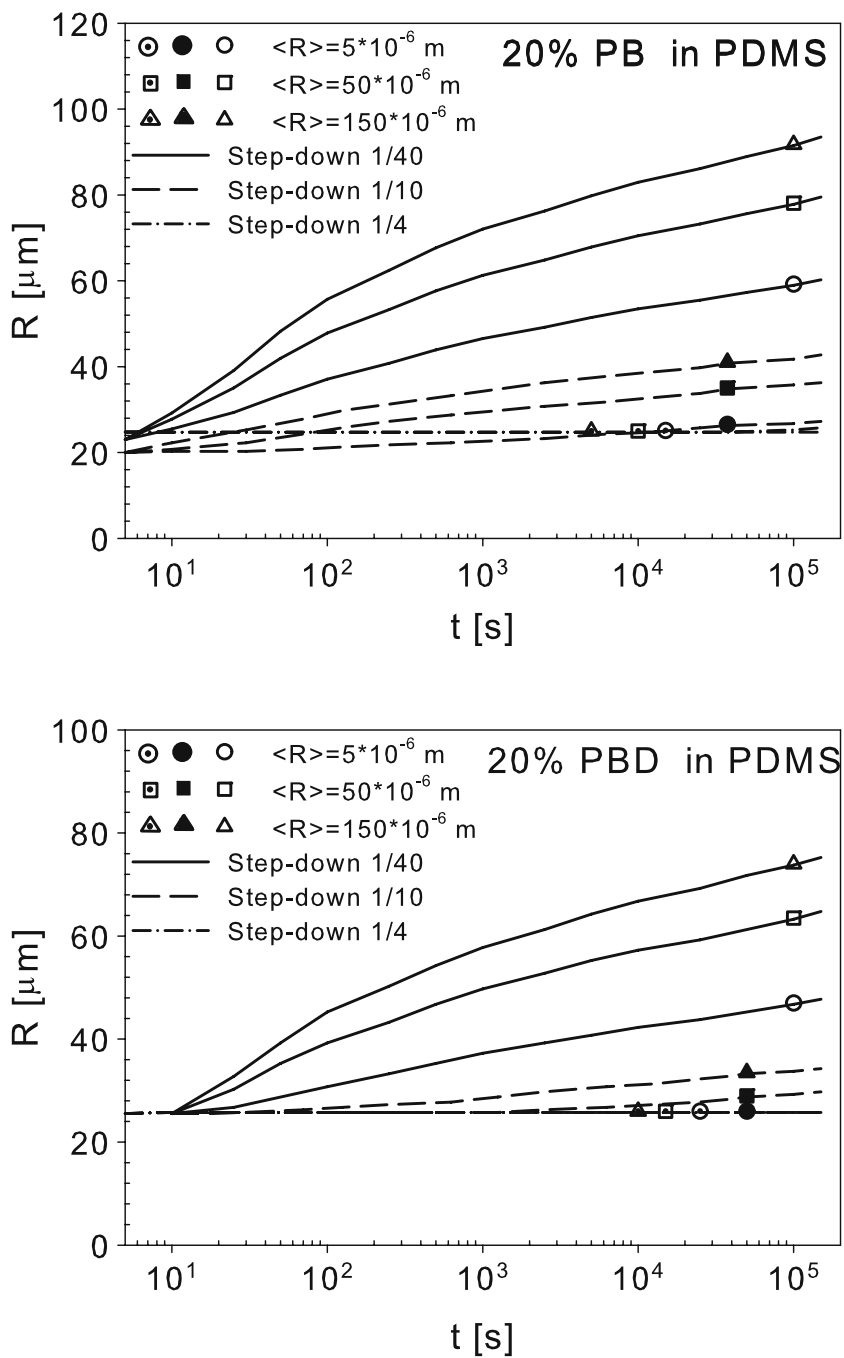

PB/PDMS system (top) and PBD/PDMS system (bottom) for concentrations of $10 \mathrm{wt} . \%$ (left) and $20 \mathrm{wt} . \%$ (right). Symbols indicate the radius used to calculate $h_{\mathrm{cr}}$ (Eq. 5), the corresponding values are reported in Table 2 

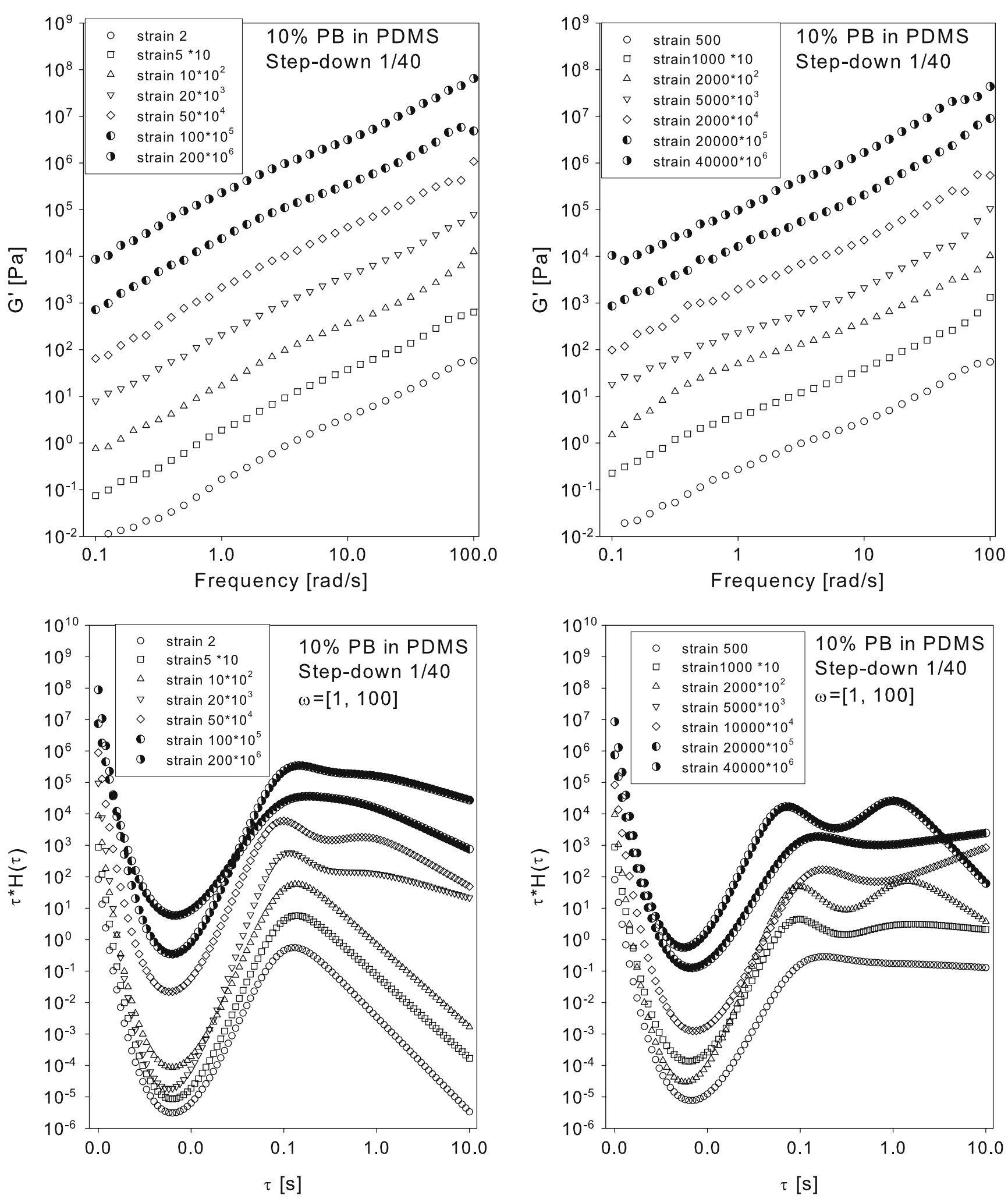

Fig. 11 Storage modulus $\mathrm{G}^{\prime}$ (top figures) and weighted relaxation spectra (bottom figures) after a step down of $1 / 40$ at different strains. For convenience, the data have been split in two plots

(strain 2 to 200 on the left, 500 to 40,000 on the right), and in both cases, the curves are shifted upwards with increasing strain units 
Substituting Eqs. 8 and 10 in Eq. 7 and expressing $Q(t), \Delta S(t)$, and $n(t)$ as functions of the volume fraction $\phi$ of the dispersed phase results in a differential equation for the time evolution of the average droplet diameter:

$\frac{d D(t)}{d t}=0.525 \phi \dot{\gamma} D(t) \exp \left[-(m D(t))^{5 / 2} \dot{\gamma}^{3 / 2}\right]$,

where $m$ is defined as:

$m=0.358\left(\frac{p}{h_{\mathrm{cr}}}\right)^{2 / 5}\left(\frac{\eta_{m}}{\sigma}\right)^{3 / 5}$

To obtain the change in drop diameter as function of the shearing time, Eq. 11 is integrated:

$\int_{m \dot{\gamma}^{3 / 5} d_{0}}^{m \dot{\gamma}^{3 / 5} d_{t}} \frac{d u(t)}{0.525 u(t) \exp \left[-u(t)^{5 / 2}\right]}=\phi \dot{\gamma} t$

where

$u(t)=m \dot{\gamma}^{3 / 5} D(t)$

Equation 13 is used to predict the time evolution of the radii, the only unknown is $h_{\mathrm{cr}}$.

\section{Coalescence results}

The $h_{\mathrm{cr}}$ values used to calculate the predictions of the PM model are chosen based on a sensitivity analysis; see Fig. 10. In the range of the average experimental radii values (see next sections), the theoretical radius predicted with the PM model is not very sensitive to changes in the value of $h_{\text {cr }}$, except for the largest stepdown in shear rate, see Fig. 10. However, we will use again the $h_{\mathrm{cr}}$ values corresponding to $R=50 * 10^{-6} \mathrm{~m}$ as a first approximation.

\section{$P B$ in $P D M S$}

Figure 11 shows the results of the dynamic measurements for different strains, for a step down in the shear rate of $1 / 40$. The slope of $G^{\prime}$ vs $\omega$ never reaches the terminal zone, i.e., becoming equal to 2 , and therefore, these results should be treated with caution. The resulting relaxation spectra are shown in Fig. 11 (bottom) for a limited frequency range of 1-100 rad/s. A second peak occurs for different strain levels. The average radii calculated from the first peak are shown in Fig. 12 (top), with the average radii corresponding to the second peaks and for all the step-downs, summarized in Table 4. The largest average radius is, as expected,
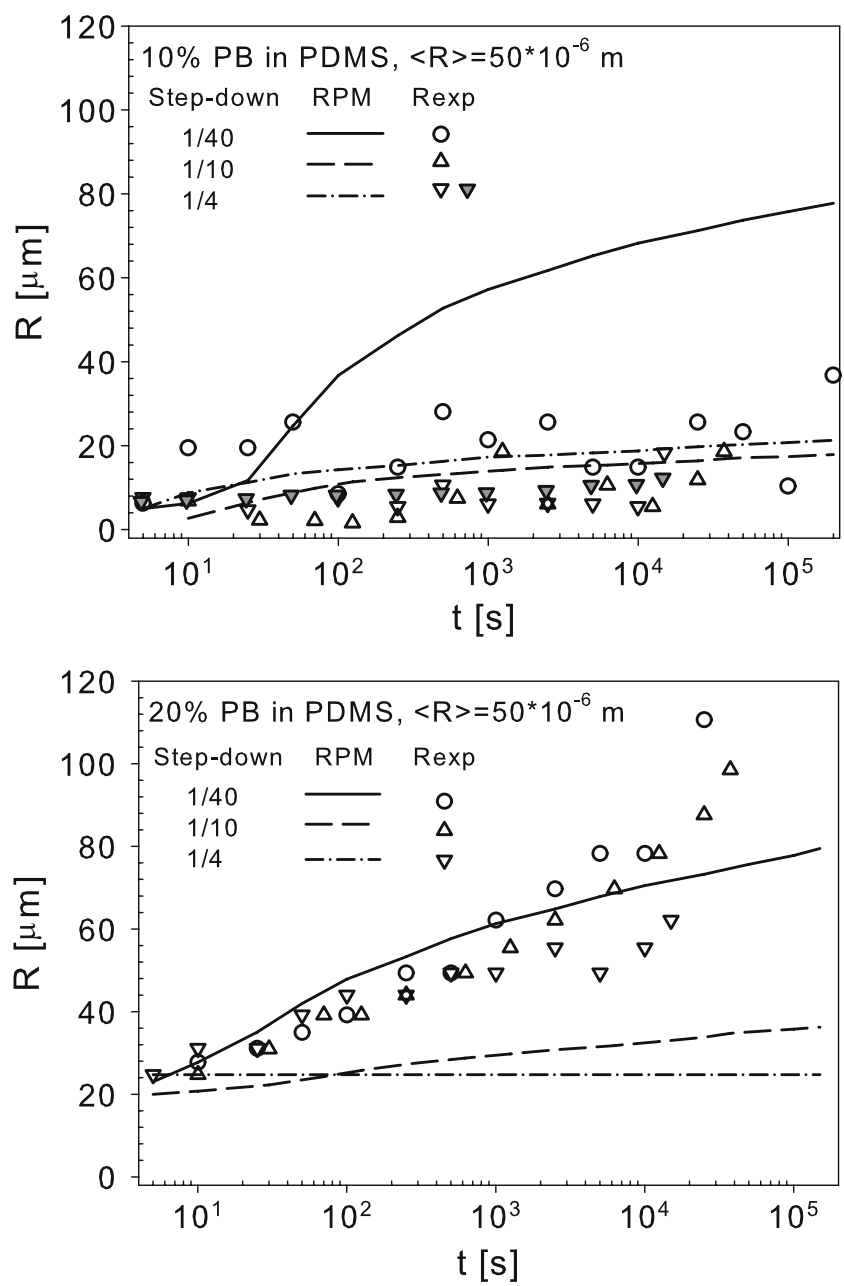

Fig. 12 Average radii calculated for the 10\% PB/PDMS system (top) and the 20\% PB/PDMS system (bottom) with Eq. 1, relaxation times obtained from weighted relaxation spectra. Lines represent the average radii calculated with Eq. 13, step-down of 1/40 (solid line), 1/10 (long dashed line), 1/4 (short dashed line). The values of $h_{\mathrm{cr}}$ used are given in the legend. Average radii calculated with OM after a step down of $1 / 4$ are also shown (gray filled circle)

found for the largest step-down in shear rate, and for all three experiments, the data show a pronounced scattering. The lines shown in Fig. 12 represent the average radius calculated with Eq. 13 and $h_{\mathrm{cr}}=2 * 10^{-8} \mathrm{~m}$ for the three step-downs of $1 / 40,1 / 10$, and $1 / 4$, respectively.

Table 4 Average radius $<\mathrm{R}>$ calculated with relaxation times from the first and second peak in the relaxation spectra, for the $10 \%$ PB in PDMS system, at step-down of $1 / 40,1.10$, and 1.4

\begin{tabular}{|c|c|c|}
\hline Step-down & $<R_{1^{\text {st }} \text { peak }}>[\mu \mathrm{m}]$ & $<R_{2^{\text {nd }}}{ }_{\text {peak }}>[\mu \mathrm{m}]$ \\
\hline$\overline{1 / 40}$ & $\sim 20$ & $100-200$ \\
\hline $1 / 10$ & $\sim 20$ & $35-170$ \\
\hline $1 / 4$ & $\sim 20$ & $30-45$ \\
\hline
\end{tabular}


The values of $<R>$ corresponding to the second peak compare reasonably well with the size of the larger droplets obtained from OM measurements for the same blend; see Fig. 4. These coalescence results also indicate the existence of a bimodal drop radius distribution. $\mathrm{OM}$ is carried out for the step down $1 / 4$, showing a smoother trend of the average radii in time compared to the rheological results, see Fig. 12 (top), and at strain units above 5,000, few larger drops with radii in the order of $<R_{2^{\text {nd }} \text { peak }}>$ are found in the majority of smaller droplets.

Figure 13 shows the elastic modulus vs the frequency (left) and the weighted relaxation spectra (right) for the $20 \%$ PB/PDMS system. For this blend, the terminal zone is reached, even at the lowest strain units.

In Fig. 12 (bottom), the average radii for the three different step downs are compared with PM model predictions. In the first $1,000 \mathrm{~s}$, the average radii seem to grow almost independently of the step down size. At longer times, the radii obtained with the larger stepdown in shear rate (1/40 and 1/10) increase sharply, while for the step-down of $1 / 4$, the growth of the average radius slows down with increasing shearing time. The steep increase in drop radius is consistent with the hysteresis results, i.e., the large average drop sizes com- pared to theoretical curves. The average radius seems to approach a constant value that is in good agreement with the average radius predicted at $\dot{\gamma}=2 \mathrm{~s}^{-1}$ by the coalescence models.

\section{$P B D$ in $P D M S$}

Also for the storage moduli measured after step downs of $1 / 40,1 / 10$, and $1 / 4$ for the 10 wt.\% PBD in PDMS blend, the terminal zone is never reached and a second peak occurs for all the strains (data not shown here). Different from the $10 \%$ PB/PDMS system, the second peak occurs at relatively long periods of time, corresponding to large radii, which are unrealistic when compared to the gap height of the cone-plate geometry. The average radii calculated from the first peak in relaxation spectrum are shown in Fig. 14 (top). For the step-down of $1 / 10$, the average radii at large strains increase quickly. The average radii obtained from $\mathrm{OM}$ when applying a step-down of $1 / 4$ are also shown in Fig. 14 (top) (filled triangle) and are in reasonable agreement with the rheological results.

For the 20-wt.\% PBD in PDMS, the elastic modulus reaches the terminal zone for all three steps down. The relaxation spectra always show one peak only.
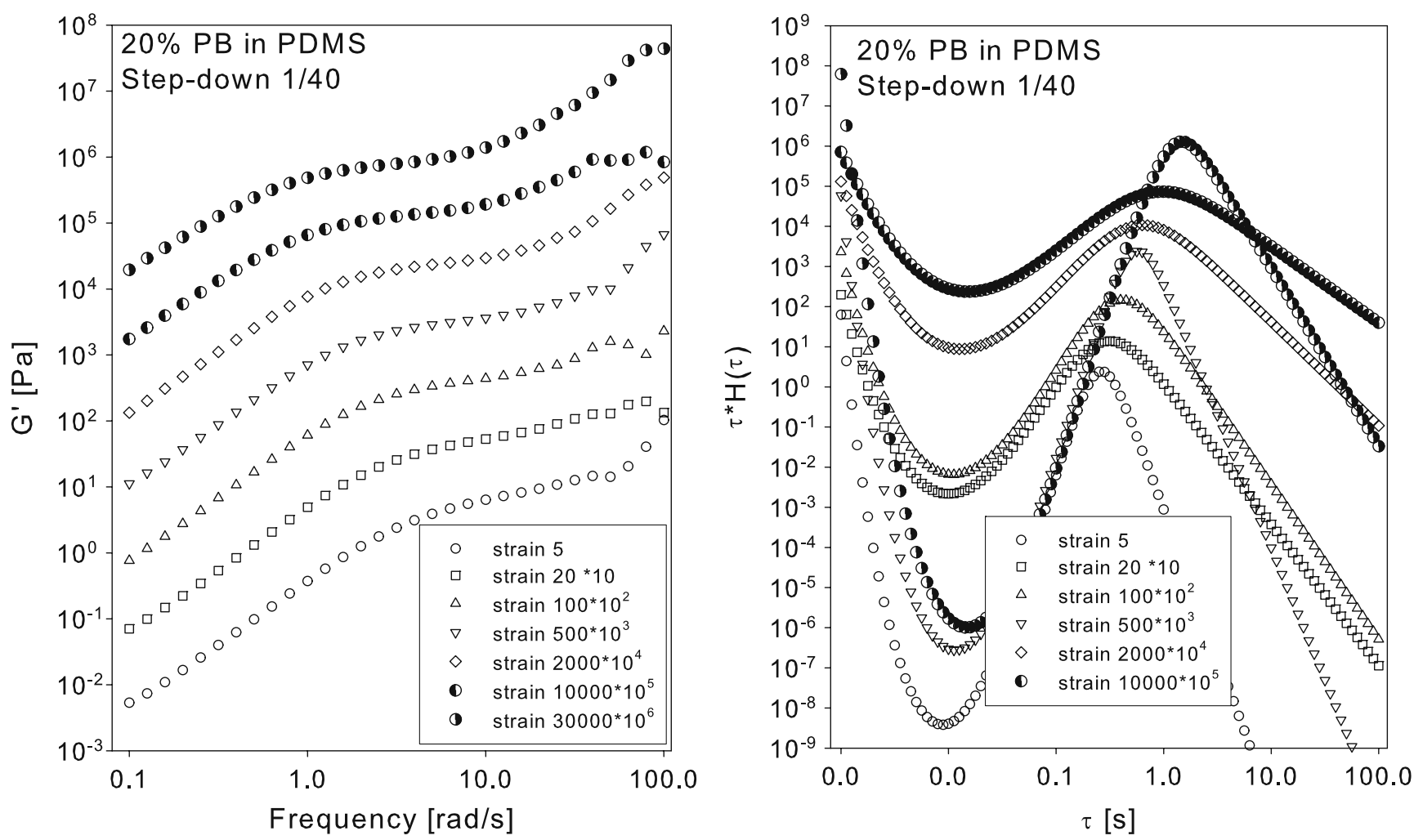

Fig. $13 \mathrm{G}^{\prime}$ vs frequency (left) and weighted relaxation spectra (right) after a step down of 1/40 to a shear rate of $0.2 \mathrm{~s}^{-1}$ 

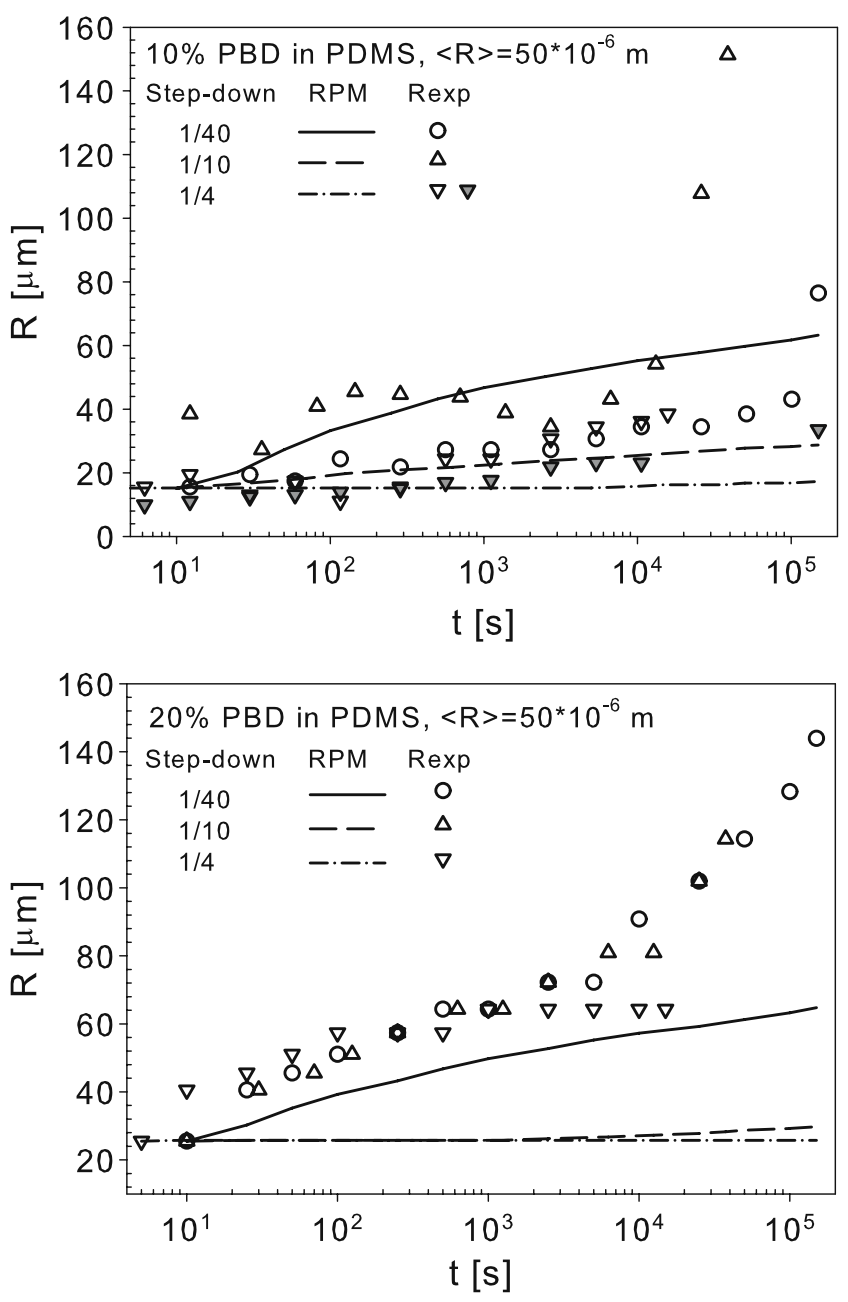

Fig. 14 As Fig. 12, now for the 10\% PBD/PDMS system (top) and the $20 \%$ PBD/PDMS system (bottom)

Figure 14 (bottom) shows the calculated average radii. Radii grow in the first 1,000 s independently of the stepdown procedure. At longer times, the average radii increase steeply for step downs of $1 / 40$ and 1/10 while they approach a "plateau" value for a step down of $1 / 4$. As shown already when investigating the hysteresis zone, the average radii are larger than the coalescence theory predictions.

\section{Conclusions}

We investigated the morphology development in two blends of partially immiscible polymers at two different concentrations by using rheological dynamic measurements and OM. The difference between the two blends is the miscibility of the components. The results are compared with predictions of relatively simple models for coalescence and breakup of droplets. The experi- mental results are rather scattered, but it can still be concluded that, for most of the cases investigated, the trends observed do not match the theoretical results. A typical feature of immiscible blends, predicted by the models and often observed in literature, is the occurrence of a hysteresis zone where the average drop size of the dispersed phase does not change with varying shear rate. This hysteresis zone is bounded between the coalescence line and the breakup line in a plot of the average radius vs shear rate. In contrast with existing literature, where the critical film thickness, $h_{\mathrm{cr}}$, is used as a fitting parameter, we calculate $h_{\text {cr }}$ from theory to predict the coalescence lines for all concentrations in a given blend. Deviations from theory, and differences between blends, become more evident in this way. For both blends studied, the experimental hysteresis zone is always narrower than predicted by breakup and coalescence theories. This is in accordance with the results of Minale et al. (1998), who measured the narrowing in hysteresis zone with increasing concentration in immiscible polymer pairs. In our experiments, the narrowing is pronounced in the (less miscible) $\mathrm{PBD} / \mathrm{PDMS}$ system, while in the (partially immiscible) PB/PDMS system, the situation is less clear since the experimental data points are also found outside the hysteresis zone, especially going beyond the breakup lines. Deviations from theory can be due to the partial miscibility of the components or to confinement effects in the coneplate configuration used. Confinement yields different structures, like ordered droplet, strings, threads, etc. Their stability depends on the degree of confinement (Son et al. 2003). After stopping the flow, and before applying oscillatory shear to determine the average drop radius, retraction of extended structures can occur or breakup of threads. In all cases, the resulting drop radii are larger than those present without confinement. Quantification clearly requires more study.

The problem of dealing with our polymer systems is that partial immiscibility itself can enhance the confinement effects mentioned. In order to illustrate this, coalescence is followed in time. Immiscible systems follow the predictions of coalescence of partially mobile systems with average radii approaching a limiting value, see Vinckier et al. (1996). In our partially immiscible systems, we observe at longer process times a rather steep and-in the experimental time scale of $10^{5} \mathrm{~s}$-an unbounded increase in average drop radius. The growth in structure far beyond its limiting value is enhanced by increasing the concentration (from $10 \%$ to $20 \%$ ) and increasing the step-down in shear rate (from $1 / 4$ to $1 / 40$ ). However, it is, maybe somewhat more surprisingly, present in both systems investigated, PBD/PDMS and PB/PDMS. Partial immiscibility results in enhanced 
dynamic coalescence, yielding larger drops that feel confinement earlier in time. Confinement effects could explain the quasi-unlimited growth in drop size measured after stopping the flow. Confinement effects, and the critical shear rate at which they occur, strongly depend on the viscosity of the components and the viscosity ratio. This could explain the unexpected differences found between the less miscible (PBD/PDMS) and the partially immiscible (PB/PDMS) systems. Clearly, to interpret data on morphology development in partially immiscible polymers from dynamic rheological measurements, applying a cone-plate geometry, should be used with caution. More quantitative studies, e.g. using combined rheological and optical experiments or applying, e.g., advanced diffuse interface modeling, that can deal with concentrated two-phase flows, should conclude whether the rheological experimental technique used could be a reliable one to approach this problem or whether it should be abandoned.

Open Access This article is distributed under the terms of the Creative Commons Attribution Noncommercial License which permits any noncommercial use, distribution, and reproduction in any medium, provided the original author(s) and source are credited.

\section{References}

Anderson D, McFadden G, Wheeler A (1998) Diffuse-interface methods in fluid mechanics. Annu Rev Fluid Mech 30: 139-165

Bruijn RD (1989) Deformation and break-up of drops in simple shear flows. Technical University of Eindhoven, Eindhoven

Cahn J, Hilliard J (1958) Free energy of a nonuniform system. I. Interfacial free energy. J Chem Phys 28(2):258-267

Cahn J, Hilliard J (1965) Phase separation by spinodal decomposition in isotropic systems. J Chem Phys 42:93-99

Chesters A (1991) The modelling of coalescence processes in fluid-liquid dispersions. Trans IChemE 69A:259-281

Elmendorp J (1986) A study on polymer blending microrheology. Technical University of Delft, Delft

Elmendorp J, van der Vegt A (1986) A study on polymer blending micro-rheology: part IV. The influence of coalescence on blend morphology origination. Polym Eng Sci 26:1332-1338

Fortelny I, Kovar J (1988) Theory of coalescence in immiscible polymer blends. Polym Compos 9:119-124

Grace H (1982) Dispersion phenomena in high viscosity immiscible fluid systems and application of static mixers as dispersion devices in such systems. Chem Eng Commun 14: 225-277

Graebling D, Gallot ABY, Muller R (1994) Dynamic viscoelastic behaviour of polymer blends in the melt: experimental results for PDMS/POE-DO, PS/PMMA and PS/PEMA blends. Eur Pol J 30(3):301-308

Graebling D, Muller R, Palierne J (1993) Linear viscoelastic behaviour of some incompatible polymer blends in melt. Interpretation of data with a model of emulsion of viscoelastic liquids. Macromolecules 26:320-329
Gramespacher H, Meissner J (1992) Interfacial tension between polymer melts measured by shear oscillations of their blends. Rheol Acta 36:1127-1141

Grizzuti N, Bifulco O (1997) Effects of coalescence and breakup on the steady-state morphology of an immiscible polymer blend in shear flow. Rheol Acta 36:406-415

Grmela M, Bousmina M, Palierne J (2001) On the rheology of immiscible blends. Rheol Acta 40(6):560-569

Honerkamp J, Weese J (1993) A nonlinear regularization method for the calculation of relaxation spectra. Rheol Acta 32: $65-73$

Jackson N, Tucker C (2003) A model for large deformation of an ellipsoidal droplet with interfacial tension. J Rheol 47: $659-682$

Janssen J (1993) Dynamics of liquid-liquid mixing. Technical University of Eindhoven, Eindhoven

Janssen J, Meijer H (1995) Dynamics of liquid-liquid mixing: a two zone model. Polym Eng Sci 35:1766-1780

Mackay G, Mason S (1963) The gravity approach and coalescence of fluid drops at liquid interfaces. J Chem Eng 41: 203-212

Migler K (2001) String formation in sheared polymer blends: coalescence, breakup, and finite size effect. Phys Rev Lett 86:1023-1026

Minale M, Mewis J, Moldenaers P (1998) Study of the morphological hysteresis in immiscible polymer blends. AIChE J 44:943-950

Minale M, Moldenaers P, Mewis J (1997) Effect of shear history on the morphology of immiscible polymer blends. Macromolecules 30:5470-5475

Palierne J (1990) Linear rheology of viscoelastic emulsions with interfacial tension. Rheol Acta 29:204-214

Pathak J, Migler K (2003) Droplet-string deformation and stability during microconfined shear flow. Langmuir 19:8667-8674

Pathak J, Davis M, Hudson S, Migler K (2002) Layered droplet microstructures in sheared emulsions: finite-size effects. J Colloid Interface Sci 255:391-402

Rusu D, Peuvrel-Disdier E (1999) In-situ characterization by small angle light scattering of the shear-induced coalescence mechanisms in immiscible polymer blends. J Rheol 43:13911409

Son Y, Martys N, Hagedorn J, Migler K (2003) Suppression of capillary instability of a polymeric thread via parallel plate confinement. Macromolecules 36:5825-5833

Takahashi Y, Kurashima N, Noda I (1994) Experimental tests of the scaling relation for textured materials in mixtures of two immiscible fluids. J Rheol 38:699-712

Taylor G (1932) The viscosity of a fluid containing small drop of another fluid. Proc R Soc Lond A 138:41-48

Tufano C, Peters G, Meijer H (2008a) Confined flow of polymer blends. Langmuir 24(9):4494-4505

Tufano C, Peters G, Anderson P, Meijer H (2008b) Transient interfacial tension of partially miscible polymers. J Colloid Interface Sci 325(1):130-140

Vananroye A, Puyvelde PV, Moldenaers P (2006) Structure development in confined polymer blends: steady-state shear flow and relaxation. Langmuir 22:2273-2280

Verdier C, Brizard M (2002) Understanding droplet coalescence and its use to estimate interfacial tension. Rheol Acta 43:514-523

Vinckier I, Moldenaers P, Mewis J (1996) Relationship between rheology and morphology of model blends in steady shear flow. J Rheol 40:613-631

Vinckier I, Moldenaers P, Terracciano A, Grizzuti N (1998) Droplet size evolution during coalescence in semiconcentrated model blends. AIChE J 44:951-958 
Von Smoluchowski M (1917) Versuch einer mathematischen theorie der koagulationskinetik kolloider Lösungen. Phys Chem 92:129-168

Yu W, Zhou C, Bousmina M (2005) Theory of morphology evolution in mixtures of viscoelastic immiscible components. J Rheol 49:215-236
Yu W, Bousmina M, Grmela M, Palierne J, Zhou C (2002) Quantitative relationship between rheology and morphology in emulsions. J Rheol 46:1381-1399

Zdravkov A, Peters G, Meijer H (2006) Film drainage and interfacial instabilities in polymeric systems with diffuse interfaces. J Colloid Interface Sci 296:86-94 